\title{
Liquid Microjunction Surface Sampling Probe Fluid Dynamics: Computational and Experimental Analysis of Coaxial Intercapillary Positioning Effects on Sample Manipulation
}

\author{
Mariam S. EINaggar, ${ }^{1}$ Charlotte Barbier, ${ }^{2}$ Gary J. Van Berkel ${ }^{1}$ \\ ${ }^{1}$ Organic and Biological Mass Spectrometry Group, Chemical Sciences Division, Oak Ridge National Laboratory, Oak Ridge, \\ TN 37831, USA \\ ${ }^{2}$ Modeling and Simulation Group, Computational Sciences and Engineering Division, Oak Ridge National Laboratory, Oak \\ Ridge, TN 37831, USA
}

\begin{abstract}
A coaxial geometry liquid microjunction surface sampling probe (LMJ-SSP) enables direct extraction of analytes from surfaces for subsequent analysis by techniques like mass spectrometry. Solution dynamics at the probe-to-sample surface interface in the LMJ-SSP has been suspected to influence sampling efficiency and dispersion but has not been rigorously investigated. The effect on flow dynamics and analyte transport to the mass spectrometer caused by coaxial retraction of the inner and outer capillaries from each other and the surface during sampling with a LMJ-SSP was investigated using computational fluid dynamics and experimentation. A transparent LMJ-SSP was constructed to provide the means for visual observation of the dynamics of the surface sampling process. Visual observation, computational fluid dynamics (CFD) analysis, and experimental results revealed that inner capillary axial retraction from the flush position relative to the outer capillary transitioned the probe from a continuous sampling and injection mode through an intermediate regime to sample plug formation mode caused by eddy currents at the sampling end of the probe. The potential for analytical implementation of these newly discovered probe operational modes is discussed.
\end{abstract}

Key words: Surface sampling probe, Fluid dynamics, Mass spectrometry, Electrospray ionization, Surface sampling

This manuscript has been authored by a contractor of the U.S. Government under contract DE-AC05-00OR22725. Accordingly, the U. S. Government retains a paid-up, nonexclusive, irrevocable, worldwide license to publish or reproduce the published form of this contribution, prepare derivative works, distribute copies to the public, and perform publicly and display publicly, or allow others to do so, for U.S. Government purposes.

Electronic supplementary material The online version of this article (doi:10.1007/s13361-011-0145-5) contains supplementary material, which is available to authorized users.

Correspondence to: Gary J. Van Berkel; e-mail: vanberkelgj@ornl.gov

\section{Introduction}

T iquid extraction-based surface sampling and ionization Lencompasses a complement of methods for acquiring analytes from surfaces under ambient conditions for subsequent analysis by MS or other techniques. [1] One implementation of these methods, a continuous-flow liquid microjunction surface sampling probe (LMJ-SSP), reconstitutes or extracts analytes from surfaces into a wall-less liquid microjunction between the sampling end of the probe and the surface. In the most common LMJ-SSP geometry, the 
extraction solvent is pumped to the surface through the annular space between two coaxial tubes at the sampling end of the probe and is then pulled into and through the inner tube to the ionization source via a self-aspirating electrospray ionization (ESI) or atmospheric pressure chemical ionization (APCI) emitter. [2-13] Recently, a non-coaxial, dual capillary, LMJ-SSP operating at nanoelectrospray flow rates was described and implemented by Roach et al. $[14,15]$

The LMJ-SSP has had two general methods of operation, viz., a discrete spot sampling method and a scanning (or imaging) method, each of which can be used either manually or as part of an automated procedure. [16] In the discrete sampling method, selected single spots from a surface are analyzed by forming a liquid microjunction separately at each of those points. The scanning or imaging method allows continuous sampling and injection of material from a surface by maintaining a liquid microjunction with the surface as the sample is moved relative to the stationary probe. Ideal operation in either method with the coaxial capillary geometry LMJ-SSP probe currently requires an empirical optimization of the relative capillary positioning. Typically, a flush alignment or slightly retracted inner capillary geometry have been the positions of choice. However, other positions might also prove beneficial. For example, in our experience, further retraction of the inner capillary creates what has appeared to be eddy flow at the sampling end of the probe. We have suspected, but have not previously reported, that this new potential operational mode might be exploited to analytical advantage.

A computational fluid dynamic (CFD) study that explains the observed optimal positioning for the capillary in the coaxial tube LMJ-SSP or uncovers other functional positions has not yet been attempted. Furthermore, no CFD studies of systems exactly like the LMJ-SSP can be found in the literature. The geometry of the probe is similar to that of the bayonet heat-exchanger, $[17$, 18] or magnetic catheter, [19] except that fluid in those devices flows from the inner tube into the annular gap formed between the two coaxial tubes. Similar to the bayonet heat-exchanger, it is expected that geometric aspect ratios and dimensionless factors, such as Reynolds numbers, can be used to characterize the majority of the properties of flow in the clearance space between the LMJ-SSP and the surface being sampled.

In this paper, we present the results from both a CFD analysis and corresponding experiments exploring various relative capillary positions in the coaxial tube LMJ-SSP. To aid in the experimental studies, we constructed a LMJ-SSP using a transparent glass outer capillary and a fused silica inner capillary that allows visual observation of the dynamics of the surface sampling process. CFD analysis revealed that inner capillary axial retraction from the flush position relative to the outer capillary transitioned the probe from a continuous sampling and injection mode to sample plug formation mode due to eddy current formation at the sampling end of the probe. These CFD analyses were validated with experimental data and visual observation of dyes within the transparent probe that had been sampled from a surface. This more thorough understanding of the LMJ-SSP fluid dynamics has revealed that at least two analytically useful modes of probe operation exist; the classic continuous sampling and injection mode as well as a plug generation mode. Intermediate operational regimes, seen here as a mix of these two modes, as well as other modes distinguished by physical methods of control are discussed.

\section{Experimental}

\section{Probe Geometry and Operation}

Figure 1a shows a schematic of the transparent LMJ-SSP apparatus. The flow of the solution in the probe follows a similar path to previously published iterations. [1-13, 16] A syringe pump (Harvard Apparatus, South Natic, MA, USA) was used to drive LC/MS grade methanol or water solutions (Fisher Scientific, Fair Lawn, NJ, USA) through the probe at $15 \mu \mathrm{L} /$ $\mathrm{min}$. The geometry of the probe, constructed of a 1/16th inch PEEK tee and fittings (Upchurch Scientific, Oak Harbor, WA, USA), directs the extraction solution from the side-arm to the surface via a glass microcapillary (Drummond Scientific Co, Broomwall, PA, USA) externally coaxial to a fused silica capillary (Polymicro Technologies Inc, Phoenix, AZ, USA), which led back through the tee. The probe outer capillary outer diameter (o.d.) and inner diameter (i.d.) were 635 and $330 \mu \mathrm{m}$, respectively. The inner capillary had a $245 \mu \mathrm{m}$ o.d. and $127 \mu \mathrm{m}$ i.d. The flowrate of solution pumped down the annulus, defined by the i.d. of the outer microcapillary and the o.d. of the fused silica capillary, was matched by the flowrate up the inner fused silica capillary controlled by aspiration from a microconcentric nebulizer (CETAC, Omaha, NE, USA), with gas flow and HV supplied by an LTQ XL linear ion trap mass spectrometer (Thermo Scientific, Waltham, MA, USA). For positive ion mode ESI, the applied voltage was $4 \mathrm{kV}$ and gas pressure was varied to match solution aspiration and pumping rates. Independent retraction and extension of the fused silica capillary was monitored at the transparent junction region, as seen in Figure 1b. A fixed union, mounted on a single axis linear translation stage (Newport Research Group, Irvine, CA, USA) provided intercapillary axial distance control to perform experiments similar to the extraction and injection computational visualizations.

An MS-2000 XYZ Stage (Applied Scientific Instrumentation Inc., Eugene, OR, USA), positioned for horizontal scanning, moved sample slides relative to the probe and controlled the stage to outer capillary distance, which defined the liquid microjunction height. Magnified views of the junction at the sample were captured through an Optem Zoom 70XL Optical System (Thales Optem Inc., Fairport, NY, USA). Optics were mounted slightly off horizontal to allow visualization of spots with an SI C400N CCD Camera (Costar Imaging, Anaheim, CA, USA), coupled to a PC through a VC 211A Actionstar A2D converter. Dual heads of a Novaflex Fiber Optic Light (World Precision Instruments, Sarasota, FL, USA) lit the probe and sample from below as well as parallel to the camera optics. 


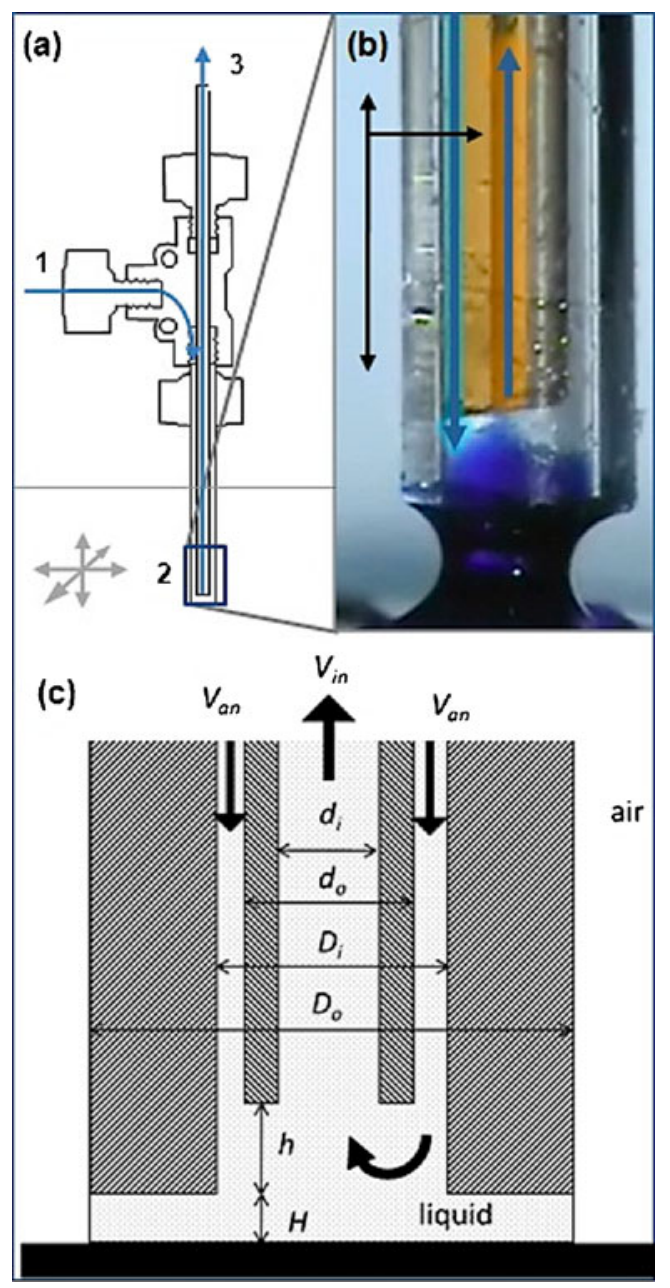

Figure 1. Diagrams and photograph showing fluid path within the LMJ-SSP. (a) Illustration of probe setup showing (1) extraction solvent pumped into the side-arm of a PEEK tee and the annular space between two coaxial capillaries toward the sampling surface to form a liquid microjunction (2), which is stabilized by a balance between the pumped flow rate and the aspirated flow rate out of the internal capillary, controlled via a pressure differential induced by ESI nebulization or a vacuum (3). (b) Photograph of the transparent sampling end of the LMJ-SSP showing a breaking junction. The position of the inner capillary can be changed via extrusion and retraction with respect to the coaxial outercapillary. (c) Schematic of junction region illustrating the junction height, $H$, inner capillary retraction distance, $h$, outer capillary diameters $\left(D_{o}\right.$ and $\left.D_{i}\right)$, inner capillary diameters $\left(d_{o}\right.$ and $d_{i}$ ), and imposed fluid velocities (annular, $V_{a n}$ and inner capillary, $V_{\text {in }}$ )

\section{Experimental Data Acquisition}

A series of red and blue ink spots from Sharpie markers (Sanford Inc., Oak Brook, IL, USA) were deposited in triplicate pairs on glass microscope slides (VWR International, West Chester, PA, USA). Injections were made by scanning the probe over the spots with the inner and outer capillary heights arranged for measurements at different liquid microjunction heights and inner capillary retractions. At low inner capillary retraction values, signal was seen at the delay time expected for flushing the probe volume to the mass spectrometer at the $15 \mu \mathrm{L} / \mathrm{min}$ flow rate of solvent through the fused silica capillary, a phenomenon referred to as "continuous sampling and injection" behavior. Greater inner capillary retraction led to concentration and mixing of analyte into a plug at the sampling end of the probe followed by junction breaking and sample being drawn into the inner capillary: these two phenomena were referred to as "plug generation" and "injection," respectively. In each case, upon junction breakage the inner capillary would be reset to zero retraction (i.e., dropped to flush) to ensure full plug injection per replicate.

Time-resolved signal traces and peaks for $\mathrm{m} / \mathrm{z}$ values of 443 and 478 , corresponding to rhodamine $6 \mathrm{G}$ and basic blue 7 from the red and blue Sharpie marker spots, respectively, were extracted from the data. The time of appearance of basic blue 7 signal depended on whether capillaries were arranged for plug generation or continuous sampling and injection. Consequently, blue dye extraction was calculated to have begun $60 \mathrm{~s}$ prior to the start time of the rhodamine $6 \mathrm{G}$ peak. Signal intensity was integrated from the calculated start time of blue dye extraction to the end of the rhodamine peak to generate the total amount of signal from any spot extraction condition set. These total signal amounts were normalized and time resolved extraction values were calculated as percentages of the total remaining sample at each time point.

\section{Computational Data Acquisition}

The flow inside the surface sampling probe was modeled based on the physical parameters indicated in Figure 1c, including the o.d. and i.d. of the outer and inner capillary ( $D_{o}, D_{i}$, and $d_{o}, d_{i}$, respectively), the inner capillary retraction from flush with the outer capillary $(h)$, the liquid microjunction probe to surface distance $(H)$, and the average solvent velocities applied at the annular probe inlet and within the inner capillary ( $V_{a n}$ and $V_{i n}$, respectively). These physical parameters determined the following dimensionless parameters: (1) the Reynolds number of the solution in the inner capillary, $R e_{i n}=V_{\text {in }} d_{i} / v$, where $v$ is the fluid kinematic viscosity, $2.045 \times 10^{-6} \mathrm{~m}^{2} / \mathrm{s}$ for $50 / 50$ methanol/water; (2) the Reynolds number of the solution in the annular region, $R e_{a n}=$ $V_{a n}\left(D_{o}-d_{i}\right) / v$; and (3) the ratios of radii, $d_{o} / d_{i}, D_{i} / d_{i}$, and $D_{o} / d_{i .}$ In this study, only physical parameters $h$ and $H$ were varied in terms of $d_{i}$. Consequently, the dimensionless parameters values remained constant: $R e_{i n}=0.78, R e_{a n}=1.2, d_{o} / d_{i}=1.65, D_{i} / d_{i}=$ 2.28, and $D_{o} / d_{i}=4.75$.

Due to these low Reynolds numbers, the flow was assumed to be axisymmetric. Direct numerical simulations were performed with CFX-12.0 (ANSYS, Inc., Canonsburg, PA, USA), a commercial, finite volume, computational fluid 
dynamics (CFD) program. A volumetric flow rate of $15 \mu \mathrm{L} /$ min was imposed on the inlet and outlet of the probe, which, given cross-sectional areas determined by relevant diameters, led to average $V_{a n}$ and $V_{i n}$ values of 3.18 and $7.90 \mathrm{~mm} /$ $\mathrm{s}$, respectively. The unsteady Navier Stokes equations in their conservation forms, assuming an incompressible fluid, constant viscosity, and neglecting gravitational force, i.e.,

$$
\begin{gathered}
\nabla \cdot U=0 \\
\frac{\partial U}{\partial t}+U \cdot \nabla U=-\frac{1}{\rho} \nabla p+v \nabla^{2} U
\end{gathered}
$$

where equations (1) and (2) are the continuity and momentum equations, respectively, $\boldsymbol{U}$ is the fluid velocity calculated at a given time and location, and $\rho$ is the density, were solved. These equations were also subject to the following boundary conditions: (1) impermeable, no slip velocity along the walls and the sample surface; and (2) a free slip wall, where velocity normal to the wall and wall shear stress are both set to zero, for the interface between liquid and air.

To simulate the extraction and pickup of a sample spot, a $1 \mu \mathrm{m}$ thick layer of a miscible "dye" was added to the surface within the calculations. Dye motion was computed by solving the following transport equation:

$$
\frac{\partial \phi}{\partial t}+\nabla \cdot(U \phi)=\nabla \cdot\left(D_{\phi} \nabla \phi\right)
$$

where $\phi$ is the dye volume fraction, $U$ is the liquid velocity and $D_{\phi}$ is the kinematic diffusivity $\left(1.225 \times 10^{-9} \mathrm{~m}^{2} / \mathrm{s}\right.$ in the current study). For this computation, at $t=0 \mathrm{~s}$, the dye was set on the sample surface and the flow at $t>0 \mathrm{~s}$ was the same as the steady flow obtained without any dye.

The calculations were run on a grid sufficiently refined to correctly simulate the flow for the Reynolds number values investigated. Supplemental Figure S1 shows an example of the mesh used, where the grid was refined in the dye layer, and near the walls. The outlet boundary was set $6.35 \mathrm{~mm}$ from the surface in order to be sufficient distance away from the junction to ensure the set boundary conditions at the outlet had no effect on the flow and diffusion could be monitored.

\section{Results and Discussion}

The LMJ-SSP arrangement used in this work is shown schematically in Figure 1a. By constructing the sampling end of the probe using glass capillaries, the actual flow dynamics of the solvent and analytes at the surface and in the transparent probe could be visually observed. The photograph in Figure 1b shows the probe forming a liquid microjunction and sampling a blue dye from the surface. The dimensions and positioning of these capillaries, as defined in
Figure 1c and described in the Experimental section and the text below, were the basis for the boundary conditions of the fluid dynamics computations.

The photographs and corresponding schematics of Figure $2 \mathrm{a}$ and $\mathrm{b}$ illustrate the different sampling and flow phenomena observed with the LMJ-SSP in the flush capillary position (at left) and with two alternative positions with the inner capillary retracted. The first case [Figure 2a(1) and $b(1)$ ] depicts the conventional optimized geometry with the capillaries positioned flush at the sampling end of the probe (i.e., $h=0$ ) and with the probe positioned approximately $20 \mu \mathrm{m}$ from the surface (i.e., $H=20 \mu \mathrm{m}$ ). This has been the typical geometry for spot sampling or imaging with the LMJ-SSP and will be referred to here as a continuous sampling and injection mode of operation. The second case [Figure $2 \mathrm{a}(2)$ and $\mathrm{b}(2)$ ] shows the probe with the same liquid microjunction thickness (i.e., probe to surface distance, $H=$ $20 \mu \mathrm{m}$ ), but with the inner capillary retracted $300 \mu \mathrm{m}$ into the outer capillary (i.e., $h=300 \mu \mathrm{m}$ ). In the third case [Figure $2 \mathrm{a}(3)$ and $\mathrm{b}(3)$ ], the inner capillary remained retracted $300 \mu \mathrm{m}$, but the liquid microjunction thickness was increased to $200 \mu \mathrm{m}$ (i.e., $H=200 \mu \mathrm{m}$ ). It should be noted that this photograph [Figure $2 b(3)$ ] indicates a potential limit of modeling, as the cylindrical approximation of the liquid microjunction differs slightly from the catenoid shape induced by surface tension as a junction is stretched before being broken. The approximation at the basis of the calculations appears sufficiently precise to account for the observed plug formation (see below).

In all three of these cases, solvent was pumped toward the surface through the capillary annulus and aspirated with a matching flow through the inner capillary to the ionization source of the mass spectrometer. When the probe geometry was set for continuous sampling and injection mode [Figure $2 \mathrm{a}(1)$ and $\mathrm{b}(1)$ ], analyte extracted from the surface was immediately aspirated into the inner capillary leading to the mass spectrometer. When the inner capillary was sufficiently retracted [Figure $2 \mathrm{a}(2)$ and $\mathrm{a}(3)$ and $2 \mathrm{~b}(2)$ and $b(3)$ ], analyte extracted from the surface remained as a plug at the sampling end of the probe. In these cases, when sufficient optical characterization using video was possible, recirculating, or eddy-like flows were observed, as depicted with arrows in the drawings in Figure $2 b(2)$ and $b(3)$. The numerical modeling results for the three geometries are shown in Figure 2c, where the solvent velocity magnitudes (based on the color gradient at the far left) are plotted on the left side of cases $2 \mathrm{c}(1), \mathrm{c}(2)$, and $\mathrm{c}(3)$, and the streamlines are plotted on the right side. Good agreement was found between the experimentally observed dye movement within the probe and calculated flow velocities and streamlines. Plug volumes, calculated based on the volume within a liquid junction and up into the sampling end of the probe, will vary based on the junction heights and inner capillary retraction. Over the range of parameters used here, that plug volume ranges between about 12 to $70 \mathrm{~nL}$. The low solvent velocities and eddy flow patterns at the end of the probe with 
(a)

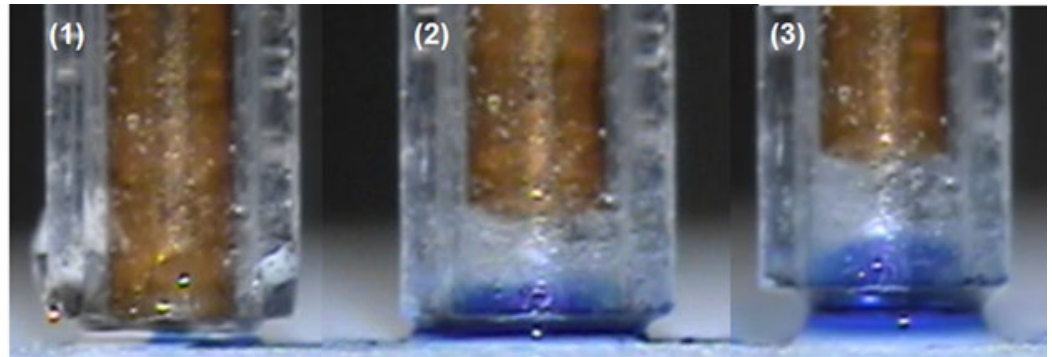

(b)

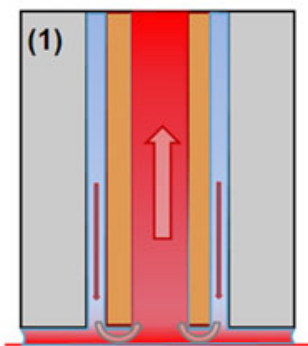

(c)

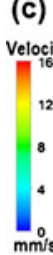

(1)

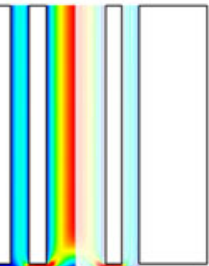

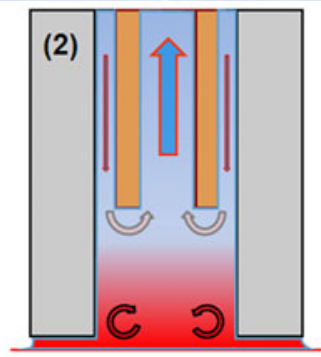

(2)

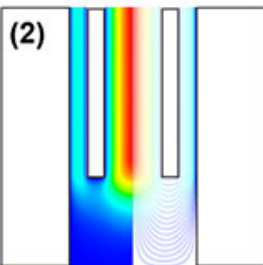

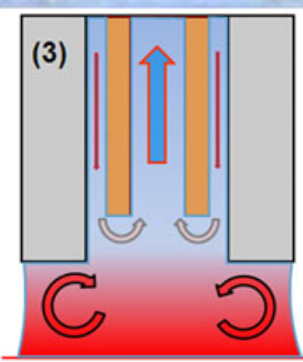

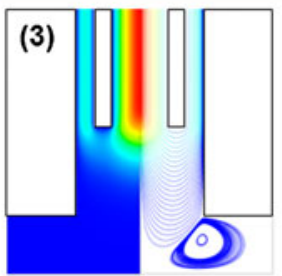

Figure 2. Illustration of observed and computationally modeled flow patterns at the sampling end of the LMJ-SSP. (a) Photographs of observed analyte extractions for a LMJ-SSP with outer capillary of $635 \mu \mathrm{m}$ o.d., and $330 \mu \mathrm{m}$ i.d., and an inner capillary of $245 \mu \mathrm{m}$ o.d. and an i.d. of $127 \mu \mathrm{m}$. Three cases are shown with capillaries positioned at (1) $H=20 \mu \mathrm{m}$ and $h=0 \mu \mathrm{m}$, (2) $H=20 \mu \mathrm{m}$ and $h=300 \mu \mathrm{m}$, and (3) $H=200 \mu \mathrm{m}$ and $h=300 \mu \mathrm{m}$. (b) Empirical flow pattern illustrations for the cases observed in (a) where the arrows indicate recirculation of analyte responsible for generation of plug despite continuous flow of solvent. (c) Corresponding simulation data with the right halves of the probes (1), (2), and (3) showing flow paths from axisymmetric ANSYS CFX computations, and the left halves of the probes showing color-coded fluid velocity, based on the scale on the far left

the inner capillary sufficiently retracted, established in the numerical modeling, made clear the mechanism for formation of the observable sample plugs in the probe.

\section{Capillary Position-Resolved Numerical Modeling}

To further characterize the effect of coaxial capillary position on the solution flow patterns and consequent extraction and aspiration of analyte from surfaces, a series of computations were run where the inner capillary retraction, $h$, was varied from 0 to $3 d_{i}$ in increments of $d_{i}$, and the junction thickness, $H$, was varied from $0.5 d_{i}$ to $1.5 d_{\mathrm{i}}$ in increments of $0.5 d_{i}$. These computations simulated the extraction of dye from a $1 \mu \mathrm{m}$ solution layer on a surface (an initial volume of $712 \mathrm{pL}$ ). Figure 3 displays the results of these computations over the matrix of conditions. The spatial distributions and normalized volume fractions of dye remaining in the probe at $60 \mathrm{~s}$ after the beginning of the sampling process are indicated using the color gradient at the bottom of the figure. The maximum fraction of dye remaining within the probe solution volume at $60 \mathrm{~s}$ was calculated to be $94.4 \%$.

Comparisons of extremes were elucidated within this matrix of conditions. Holding the junction height, $H$, constant, i.e., looking across rows of the images in Figure 3, inner capillary retraction can be seen as the effective cause of sample plug generation. The cases depicted with the maximum inner capillary retraction (Figure $3 \mathrm{~d}, \mathrm{~h}$, and $\mathrm{l}$ ) all show plug formation within the time period of the simulation. In these cases, the area between the outer capillary and the surface remains unimpinged upon by solvent flow-lines, as seen by the solid, consistent color. A more subtle effect was seen when holding the inner capillary retraction constant, i.e., varying only the distance the probe was positioned above the surface. Comparing images in one column of the figure - in the case of $h=0$, Figure $3 \mathrm{a}$, e, and $\mathrm{i}$ - the largest junction (i.e., Figure $3 \mathrm{i}, H=$ $1.5 d_{\mathrm{i}}$ ) resulted in nearly complete $(99.2 \%)$ pickup and injection of the dye over the course of $60 \mathrm{~s}$. When the liquid junction distance was small (i.e., Figure $3 \mathrm{a}, H=0.5 d_{\mathrm{i}}$,), residual dye was observed on the edges of the volume closest to the outer diameter of the outer capillary. Residual dye in the junction after $60 \mathrm{~s}$ despite lack of generation of a distinct plug was because the streamlines of the flow were more compressed with the small junction height. This caused recirculating eddies along the outer edge of the junction volume. When the $h / d_{\mathrm{i}}$ ratio was increased, the recirculation flow was formed higher in the outer capillary and, thus, more dye was left within the domain shown. When the $H / d_{\mathrm{i}}$ ratio was increased, the recirculation of solution below the outer capillary also increased and all the dye could be removed from the surface. 


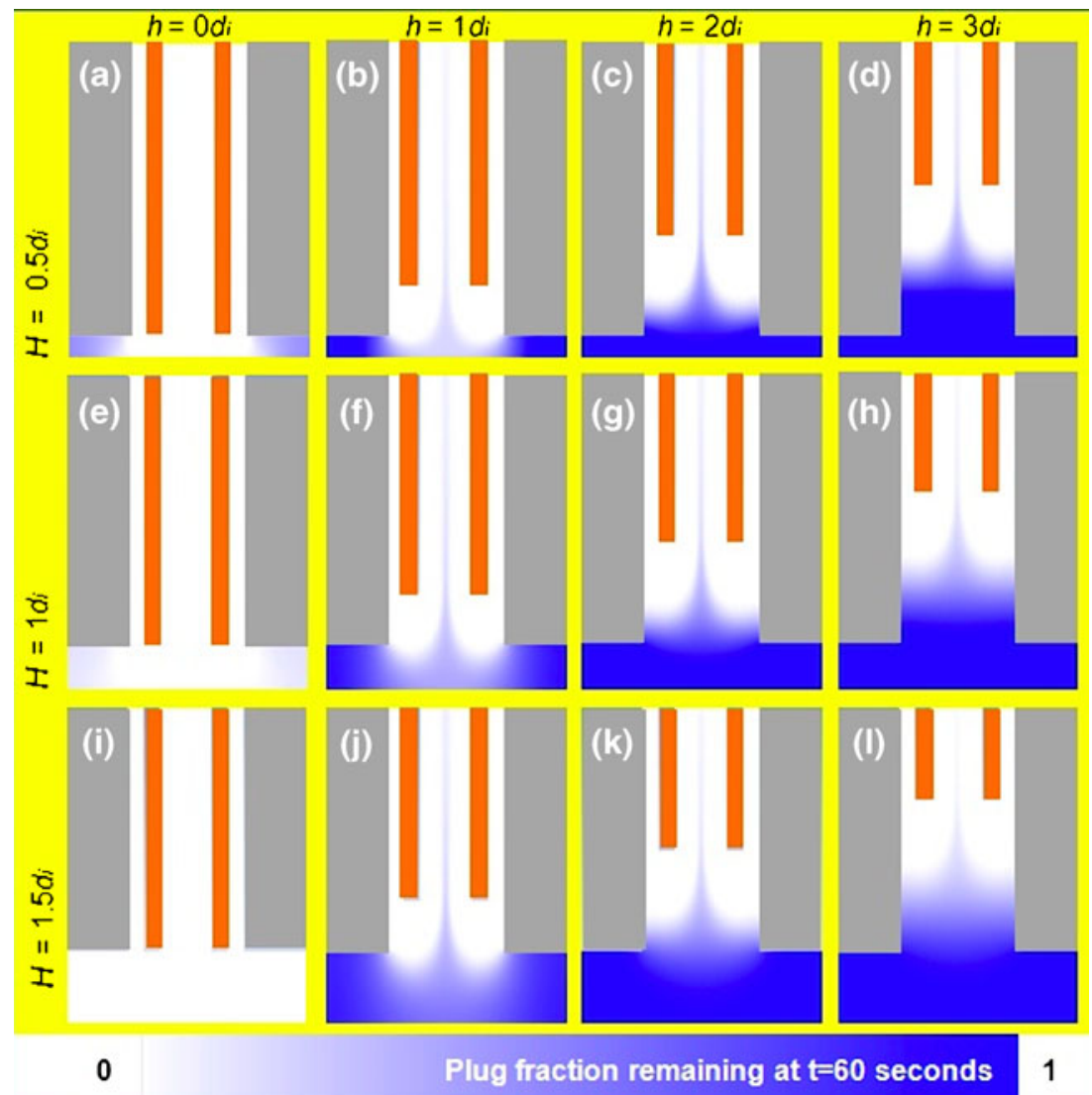

Figure 3. Discrete numerical simulations for the sampling process, varying both liquid junction height, $H$, and inner capillary retraction, $h$, in terms of inner capillary inner diameter, $d_{i}$. The matrix of results shows changes in parameter $h$ across rows and changes of parameter $H$ down columns. Grey walls correspond to the glass outer capillary and the orange walls correspond to the fused silica inner capillary. Computed data are shown that correspond to $60 \mathrm{~s}$ of flow based on the geometrically proscribed velocity profiles, where the fraction of sample remaining after the initiation of the surface sampling process within the probe is indicated by the color of the fluid in the images shown

Solvent velocity magnitudes and the streamlines for each of the cases shown in Figure 3 are shown in Figure 4 to further elucidate the flow paths leading to plug generation or injection. At larger $h / d_{\mathrm{i}}$ ratios there was less flow near the surface and consequently dye must diffuse into the junction before being convected by flow into the inner capillary. This means that with an increasing $H / d_{i}$ ratio, a larger fraction of the dye will remain within the junction volume and recirculating eddy. This diffusive effect appears to become dominant between $h / d_{i}=0$ and $h / d_{\mathrm{i}}=1$, though these calculations are also specific to the kinematic diffusivity of the solvent $\left[D_{\phi}\right.$ in this case was $1.225 \times 10^{-9} \mathrm{~m}^{2} / \mathrm{s}$, simulating $50: 50$ (vol/vol) methanol/water]. Increasing diffusivity leads to a larger fraction of the dye being aspirated due to the dye diffusing into the areas where flow lines sweep solvent into the inner capillary.

\section{Capillary Position and Time Resolved Experimental Results}

To experimentally validate the theories abstracted from the simulated data and visual observations above, the LMJ-SSP was set up to run under the same conditions as in the computational model. The experimental setup shown in Figure 5a depicts triplicate replicates of spots for analysis, where individual surface scans, corresponding to individual data points, are represented by the arrows. For a single data point, a liquid microjunction was formed between the probe and a blank glass surface with junction height and inner capillary retraction values set to correspond to the desired conditions of $H$ and $h$. The probe was then scanned onto a spot of the blue Sharpie dye and allowed to extract analyte for $60 \mathrm{~s}$. Subsequently, the probe was scanned onto a spot of red Sharpie dye, where the probe was allowed to extract analyte for $12 \mathrm{~s}$ before being retracted from the surface, breaking the junction. The inner capillary was immediately dropped to the flush position $(h=0)$ at which point any solution within the inter-capillary volume was injected if not already aspirated into the inner capillary.

Qualitatively, the differences in operation between continuous sampling and injection mode and plug formation mode followed by injection were visible over the course of the experiment, as is shown in Figure $5 \mathrm{~b}$ and c. For the photographs shown in Figure $5 \mathrm{~b}$, the inner capillary 


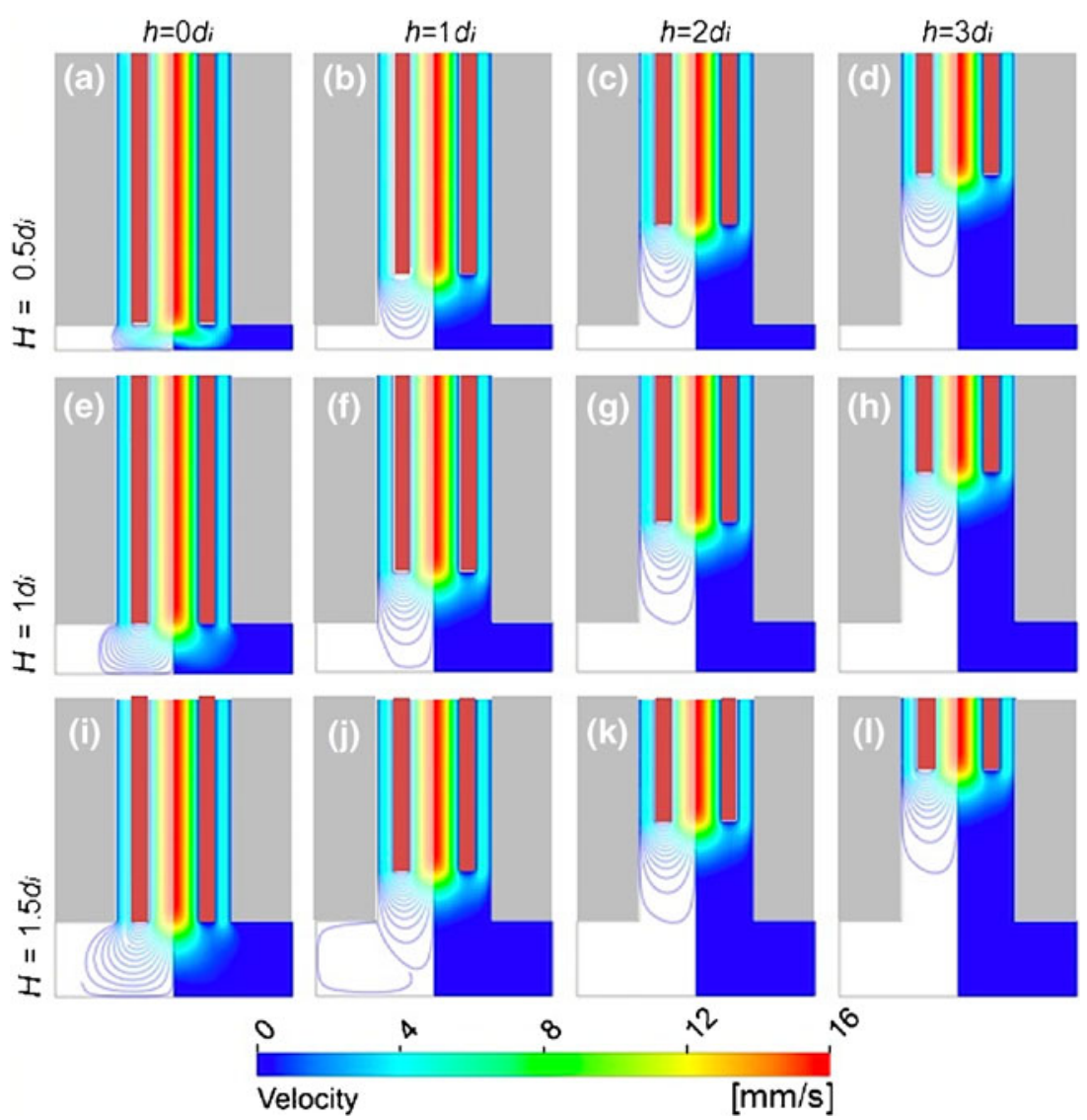

Figure 4. Computationally modeled flow patterns at the sampling end of the LMJ-SSP. Discrete numerical simulation data of fluid velocities and streamlines based on changing the liquid junction height, $H$, and the inner capillary retraction, $h$, in terms of inner capillary inner diameter, $d_{i}$. Calculated data are shown in where the right halves of probes show flow paths from axisymmetric ANSYS CFX computations with boundary conditions from Figure 3, and the left halves of the probes show velocity, color coded based on the scale on the bottom. Grey walls correspond to the glass outer capillary and the orange walls correspond to the fused silica inner capillary

retraction $h$ was zero, and the junction height $H$ was $0.5 d_{\mathrm{i}}$. At the beginning of extraction (far left image), the blue dye was clearly visible being aspirated into the inner capillary. This continued over time (second image from left), as the extraction was completed over the course of $60 \mathrm{~s}$. By the time the capillary was scanned to the red spot, the red dye had a visible injection front (shown in the third image). This visible front is similar to the blue front seen in the initial spot extraction, as no mixing between red and blue took place. Upon finishing the injection, the remainder of the red dye was aspirated, as seen in the far right image. For the photographs shown in Figure 5c, the inner capillary retraction was increased to $3 d_{i}$, as is visible in the far left image. Extraction with a junction of $H=0.5 d_{i}$ yields a blue plug. None of the blue dye moved beyond the eddy layer in the bottom fraction of the probe (second image from left). Upon scanning over the red spot, this blue dye mixed with the red dye, generating a purple plug in the same location of the probe (third image). Lifting the probe from the surface and breaking the junction, as shown in the far right image, generated a fluidic disturbance that caused the plug to take up a slightly larger volume of the probe, as there was no longer solution between the probe and the surface. However, the analyte plug remained at the sampling end of the probe until the inner capillary was moved to the flush position.

These sampling and injection phenomena were monitored using the extracted ion current profiles for the primary components of the red and blue dyes detected in the mass spectrometer at $\mathrm{m} / \mathrm{z} 443$ and 478 , respectively. Triplicate replicates of the sampling experiments with $h=0 d_{i}$ and $h=$ $3 d_{i}$ are shown in Figure $5 \mathrm{~d}$ and e, respectively. In Figure $5 \mathrm{~d}$, the peak corresponding to the extraction of blue dye appears one min prior to the peak corresponding to the red dye. This is in stark contrast to the red and blue dye peaks in Figure 5e, which appear simultaneously. That is, despite scanning over the spatially separated blue and red dye spots sequentially, the mass spectral signals corresponding to the different dyes appear concurrently instead of consecutively. This shows that the sampled analytes were spatially concentrated and mixed before injection.

Using the signal from the red dye as a time point for alignment, the blue dye signal intensity for an individual 


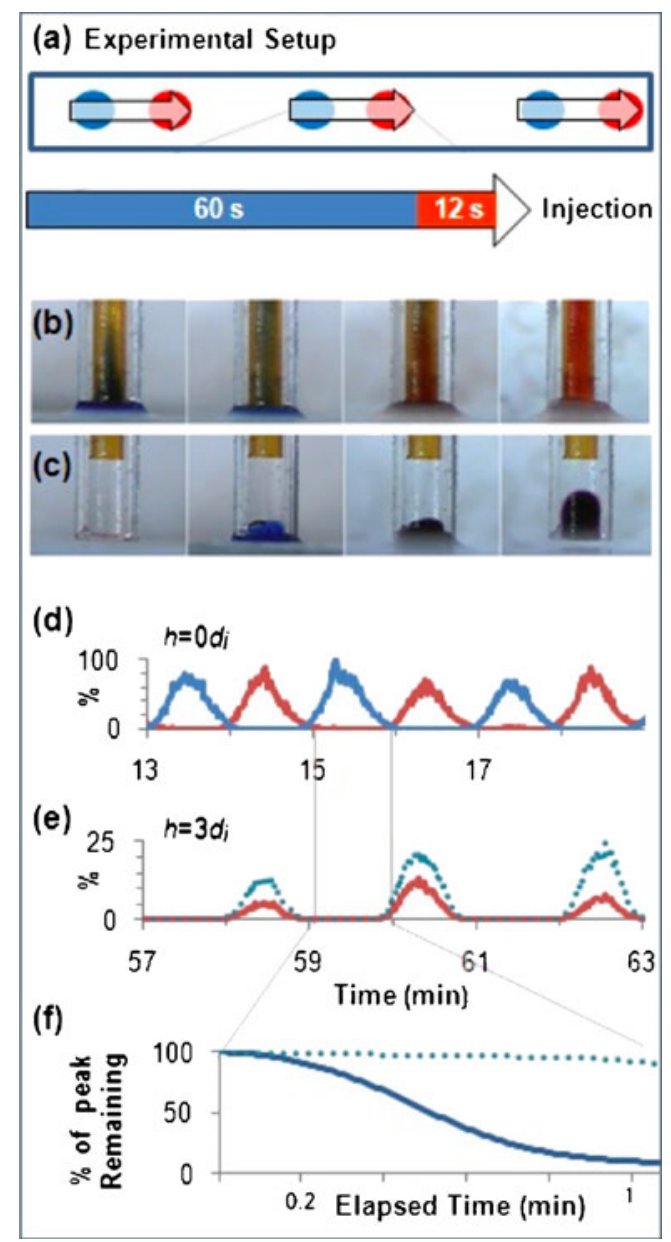

Figure 5. Triplicate paired-spot scanning experimental setup and exemplar data. Each experimental replicate consisted of a $60 \mathrm{~s}$ extraction on a blue spot, followed by a $12 \mathrm{~s}$ extraction on a red spot, followed by an injection after breaking the liquid microjunction to the surface, as shown in panel (a). Panels (b) and (c) show a series of photographs for $h=0$ and $h=3 d_{i}$, respectively, with fixed $H=0.5 d_{i}$. The time resolved mass spectral signal from the continuously extracted or injected plug of blue $(\mathrm{m} / \mathrm{z} 478)$ and red $(\mathrm{m} / \mathrm{z}$ 443) spots sampled under conditions of $h=0$ and $h=3 d_{i}$ are plotted in (d) and (e), respectively. (f) These data were aligned, averaged, and normalized for the two experimental conditions in order to plot the time resolved percentage of the sample plug remaining in the probe for conditions $h=0$ (solid dark blue plot) and $h=3 d_{i}$ (dotted light blue plot)

replicates could be integrated and normalized, with triplicate measurements averaged to generate time-resolved extraction percentages for individual conditions, as shown in Figure $5 \mathrm{f}$. The dotted blue line corresponds to the signal seen with $h=$ $3 d_{i}$, and the solid blue line corresponds to the signal seen with $h=0 d_{i}$. The percent of the peak remaining at $1 \mathrm{~min}$ in the former case was nearly $100 \%$ due to plug generation. The plot for the latter case (the solid blue line) shows nearly complete extraction of blue dye. The shape of this plot indicates some effects of diffusion, with the small amount remaining at $1 \mathrm{~min}$ corresponding to the previously simulated data (Figure 3a) with near complete extraction within the same amount of time, in the flow line accessible areas (Figure 4a), but not complete extraction.

\section{Comparative Theoretical and Experimental Analysis}

The subset of experiments depicted in Figure 5 was completed for the total array of conditions of the previous capillary position resolved numerical modeling. Both the computational and experimental results are depicted in the plots shown in Figure 6, where panel a shows computational data and panel $b$ shows experimental data that were generated in the same manner as the data displayed in Figure 5f. For all the plots, the black lines correspond to junction heights, $H$, of $0.5 d_{\mathrm{i}}$, and the dark and light grey lines to $1.0 d_{\mathrm{i}}$ and $1.5 d_{\mathrm{i}}$, respectively. Numbered plots in both panels correspond to inner capillary retractions of (1) $h=0 d_{\mathrm{i}}$, (2) $h=1.0 d_{\mathrm{i}}$, (3) $h=2.0 d_{\mathrm{i}}$, and (4) $h=3.0 d_{\mathrm{i}}$. The inset plots in the computed data in panel (a) show the time window over which the first $10 \%$ of the dye was injected, which ranged from under $1 \mathrm{~s}$ (1) to over $1 \mathrm{~min}$ (4).

The time-resolved data for individual replicates was aligned, integrated, normalized, and averaged $(n=3)$ as described in the Experimental section. The resulting experimental data were comparable to the time-resolved data generated by the simulations performed using the same $h$ and $H$, values varied in terms of the constant inner capillary diameter, $d_{i}$. The steep slopes and small fractions of plugs remaining after $1 \mathrm{~min}$, both in the calculated and observed data shown in (1), were consistent with a continuous sampling and injection mode, where there was no delay in sample aspiration into and through the probe. In the case of (2), where $h=1.0 d_{\mathrm{i}}$, the plotted experimental data (panel a) exceeds the extraction fractions for the numerically simulated data shown in (panel b) visible in the relative amounts remaining in all junction height based curves. This difference between simulated and experimental data may have resulted from higher levels of extraction caused by the physical effects of probe movement onto the spots, which was not taken into account by the simulation. Similar to the significant difference between continuous sampling and injection and plug generation mode extractions seen previously (i.e., Figure 5f), there was an obvious difference between time resolved integrated signal for the low and high $h$ values, both in the computed and experimental data. Inset plots depicting a magnified view of the simulated extraction of the first $10 \%$ of the dye (panel a) also show similarities between data from numerical simulations and the correlated experiments. Despite decay-like appearance in the longer length scale, a sigmoidal curve was seen in all cases (panel b).

Data from the numerical simulations panel (a) show extraction in cases of $h=1.0 d_{\mathrm{i}}$ and $2.0 d_{\mathrm{i}}$, as intermediates between $h=0 d_{\mathrm{i}}$ and $h=3.0 d_{\mathrm{i}}$, which would appear to indicate a linear dependence on inner capillary retraction. However, experimental data (panel b) show either immediate extraction to near completion, (plots 1, 2), or a plug generation 


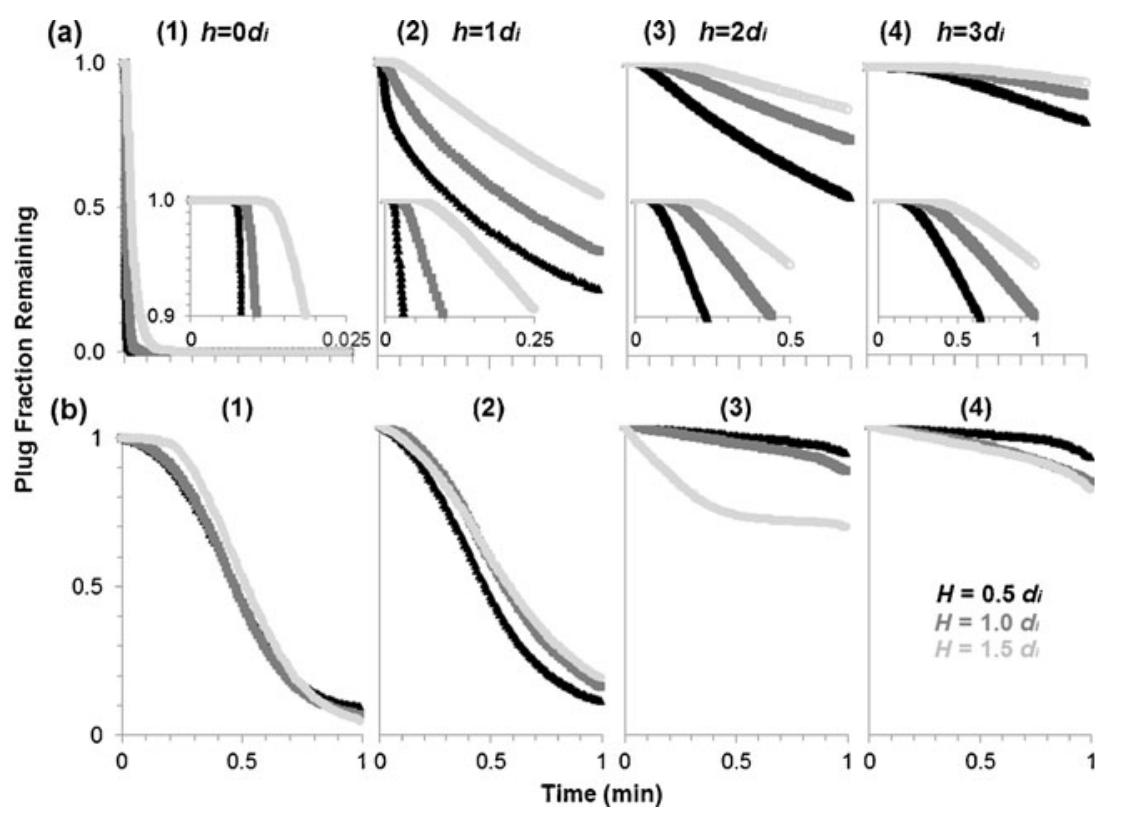

Figure 6. Plotted fractions of analyte remaining during $60 \mathrm{~s}$ of extraction with the LMJ-SSP. Computed data (a) and corresponding experimental data (b) are shown, where the three different lines plotted represent results for different values of $H$. Black line data, $H=0.5 d_{i}$; dark grey line data, $H=d_{i}$; and light grey data, $H=1.5 d_{i}$. Results for inner capillary retraction simulations and experiments are shown for $h=0 d_{i}(1), h=1 d_{i}(2), h=2 d_{i}$ (3), or $h=3 d_{i}$ (4). Inset plots for the computed data (a), show the extraction of the first $10 \%$ of the peak fractions

from which little extraction can take place until injection (plots 3, 4). Raw experimental data for the triplicate experiments in these cases included peaks that looked like combinations of peaks similar to those shown in Figure 5d and e. In the case of an inner capillary retraction length of $2 d_{i}$ and a junction height of $1.5 d_{\mathrm{i}}$ (i.e., the light grey curve in Figure $6 \mathrm{~b}$, plot 3 ) the raw data consisted of two pairs of peaks looking identical to those shown in Figure 5e (plug injection-like), and one pair looking like the addition of peaks in Figure $5 \mathrm{~d}$ and e. For this reason, the average plug fraction remaining appears intermediate to the continuous sampling and injection and plug generation thresholds.

Both in the computational and experimental data, the effects of inner probe retraction dominate over the effects of junction height. This is visible in that all curves in any individual plot in Figure 6 appear more similar to each other (e.g., the black, dark grey, and light grey curves in plot 4) than to other curves of the same color (e.g., black curves across panel b). Experimentally (panel b), the effects of junction height appear more significant near the threshold between continuous sampling and injection mode and plug generation mode, as seen looking at the difference between $h=1.0 d_{\mathrm{i}}$ and $2.0 d_{\mathrm{i}}$, (i.e., plots 2 and 3 ), where the diffusion and convection of dye into the streamlines of flow can lead to aspiration of dye instead of plug generation. This difference between the experimental and calculated data could come from subtle differences between the modeled and actual probe used. Calculations for the plug fractions remaining were made monitoring the flux $6.53 \mathrm{~mm}$ from the sampling end of the inner capillary shown in the calculation domain image, depicted on the right side of Supplementary Figure S1. In the experiments, however, measurements were taken after the analyte traveled $310 \mathrm{~mm}$ away from the probing end of the inner capillary. The calculations did not include the diffusion effects caused by laminar pipe flow in the inner capillary, which would yield broadening of peaks by factor of approximately 1.2, assuming Poiseuille flow over the length of the longer $310 \mathrm{~mm}$ inner capillary. However, this broadening would equally apply to all calculated values and, consequently, not change the qualitative comparisons between experimental and calculated data.

\section{Conclusions}

In this investigation of flow dynamics, CFD calculations, experiments, and visual observation with a transparent probe were used to understand the classic coaxial tube LMJ-SSP operational mode and discover and characterize new operational modes though the variation of inner capillary retraction distance and the height of the liquid microjunction above a surface. These studies showed that inner capillary axial retraction from the flush position relative to the outer capillary transitioned the probe from a continuous sampling and injection mode (classic operation) through an intermediate regime to a sample plug formation mode caused by eddy currents at the sampling end of the probe. This new plug formation mode, and variations thereof, demonstrated by the ability to pickup, hold, and mix multiple samples within a nanoliter-scale volume at the sampling end of the probe, prefigures many analytical opportunities. For example, this 
plug formation mode might be exploited to not only extract and analyze sample from a surface, but also concentrate and manipulate plugs in order to run chemical reactions of species in nanoscale volumes, for extended periods of time (several minutes or more) prior to injecting the sample for analysis. Future studies will be aimed at exploiting this capability to extract, mix, and concentrate multiple analytes within the LMJSSP for analytical benefit.

\section{Acknowledgments}

Dr. Vilmos Kertesz (ORNL) is thanked for critical review of this manuscript. The authors acknowledge support for this work by the Division of Chemical Sciences, Geosciences, and Biosciences, Office of Basic Energy Sciences, United States Department of Energy. ORNL is managed by UTBattelle, LLC for the U.S. Department of Energy under contract DE-AC05-00OR22725.

\section{References}

1. Van Berkel, G.J., Pasilis, S.P., Ovchinnikova, O.: Established and Emerging Atmospheric Pressure Surface Sampling/Ionization Techniques for Mass Spectrometry. J. Mass Spectrom. 43, 1161-1180 (2008)

2. Wachs, T., Henion, J.: Electrospray Device for Coupling Microscale Separations and Other Miniaturized Devices with Electrospray Mass Spectrometry. Anal. Chem. 73, 632-638 (2001)

3. Van Berkel, G.J., Sanchez, A.D., Quirke, J.M.E.: Thin-Layer Chromatography and Electrospray Mass Spectrometry Coupled using a Surface Sampling Probe. Anal. Chem. 74, 6216-6223 (2002)

4. Wachs, T., Henion, J.: A Device for Automated Direct Sampling and Quantitation from Solid-Phase Sorbent Extraction Cards by Electrospray Tandem Mass Spectrometry. Anal. Chem. 75, 1769-1775 (2003)

5. Ford, M.J., Van Berkel, G.J.: An Improved Thin-Layer Chromatography/Mass Spectrometry Coupling Using a Surface Sampling Probe Electrospray Ion Trap System. Rapid Commun. Mass Spectrom. 18, 1303-1309 (2004)

6. Ford, M.J., Kertesz, V., Van Berkel, G.J.: Thin Layer Chromatography/ Electrospray Ionization Triple Quadrupole Linear Ion Trap Mass Spectrometry System: Analysis of Rhodamine Dyes Separated on Reversed-Phase C8 Plates. J. Mass Spectrom. 40, 866-875 (2005)
7. Ford, M.J., Deibel, M.A., Tomkins, B.A., Van Berkel, G.J.: Quantitative Thin-Layer Chromatography/Mass Spectrometry Analysis of Caffeine Using a Surface Sampling Probe Electrospray Ionization Tandem Mass Spectrometry System. Anal. Chem. 77, 4385-4389 (2005)

8. Asano, K.G., Ford, M.J., Tomkins, B.A., Van Berkel, G.J.: SelfAspirating Atmospheric Pressure Chemical Ionization Source for Direct Sampling of Analytes on Surfaces and in Liquid Solutions. Rapid Commun. Mass Spectrom. 19, 2305-2312 (2005)

9. Kertesz, V., Ford, M.J., Van Berkel, G.J.: Automation of a Surface Sampling Probe/Electrospray Mass Spectrometry System. Anal. Chem. 77, 7183-7189 (2005)

10. Van Berkel, G.J., Ford, M.J., Doktycz, M.J., Kennel, S.J.: Evaluation of a Surface Sampling Probe Electrospray Mass Spectrometry System for the Analysis of Surface Deposited and Affinity Captured Proteins. Rapid Commun. Mass Spectrom. 20, 1144-1152 (2006)

11. Van Berkel, G.J., Kertesz, V., Koeplinger, K.A., Vavrek, M., Kong, A. T.: Liquid Micro-Junction Surface Sampling Probe Electrospray Mass Spectrometry for Detection of Drugs and Metabolites in Thin Tissue Sections. J. Mass Spectrom. 43, 500-508 (2008)

12. Emory, J.F., Walworth, M.J.: Van Berkel. G. J.; Schulz, M.; Minarik, S.: Direct Analysis of Reversed-Phase High Performance Thin Layer Chromatography Separated Tryptic Protein Digests Using a Liquid Microjunction Surface Sampling Probe/Electrospray Ionization Mass Spectrometry System. Eur. J. Mass Spectrom. 16, 21-33 (2010)

13. Walworth, M.J., Stankovich, J.J., Van Berkel, G.J., Schulz, M., Minarik, S., Nichols, J., Reich, E.: Hydrophobic Treatment Enabling Analysis of Wettable Surafaces using A Liquid Microjunction Surface Sampling Probe/Electrospray Ionization-Mass Spectrometry System. Anal. Chem. 83, 591-597 (2011)

14. Roach, P.J., Laskin, J., Laskin, A.: Nanospray Desorption Electrospray Ionization: An Ambient Method for Liquid-Extraction Surface Sampling in Mass Spectrometry. Analyst 135, 2233-2236 (2010)

15. Roach, P.J., Laskin, J., Laskin, A.: Molecular Characterization of Organic Aerosols using Nanospray-Desorption/Electrospray IonizationMass Spectrometry. Anal. Chem. 82, 7979-7986 (2010)

16. Van Berkel, G.J., Kertesz, V., King, R.C.: High Throughput Mode Liquid Microjunction Surface Sampling Probe. Anal. Chem. 81, 70967101 (2009)

17. Minhas, H., Lock, G.S.H., Wu, M.: Flow Characteristics of an Air Filled Bayonet Tube under Laminar Conditions. Int. J. Heat and Fluid Flow. 16, 186-193 (1995)

18. Minhas, H., Lock, G.S.H.: Laminar-Turbulent Transition in a Bayonet Tube. Int. J. Heat and Fluid Flow. 17, 102-107 (1996)

19. Humphrey, J., Ferrante, E., Rosales, L., Gillies, G.: Flow and Heat Transfer in the End Space of a Magnetically-Guided Concentric Tubes Catheter. Proceedings of the 9th AIAA/ASME Joint Thermophysics and Heat Transfer Conference. Collected Technical Papers. San Francisco, CA. June 5, 2006 